dangerous environments, with a high risk of injury due to the lack of control measures in risk factors.

Objective To describe the health and working conditions of waste pickers worldwide, through a review of scientific articles published between the years 1999 to 2019 .

Methods A scoping review was carried out, where scientific articles were identified by manual bibliographic search in PubMed, Taylor and Francis, ScienceDirect and SAGE. The Mendeley bibliographic manager was used to refine the articles. The review of the records was based on: type of document, reading of titles, abstracts, methodology and results. Inclusion and exclusion criteria were taken into account for the selection of articles.

Results Eight-eight studies were included that met the inclusion criteria. Most of the studies analyzed used a qualitative approach, where questionnaires, interviews and focus groups were applied. Waste pickers' working conditions were mainly associated with situations present in the work environment, non-working conditions are largely associated with their economic, family and interpersonal relationships. The individual conditions with the highest incidence are associated with gender, age and education. The health conditions are related to the physical capacity of the waste picker to carry out the work, the consumption of tobacco and other substances and the prevalence of some diseases such as tuberculosis and skin rashes.

Conclusion It is important to expose to the academic and governmental community the problems that waste pickers face in the development of their work in order to establish tools to improve the health and working conditions of this vulnerable population.

\section{P-247 WORK-RELATED DISEASES AND INJURIES AMONG CULTIVATED AGRICULTURISTS IN THAILAND}

${ }^{1}$ Sunisa Chaiklieng, Pornnapa Suggaravetsiri. ${ }^{1}$ Faculty of Public Health Khon Kaen University, Thailand

\subsection{6/OEM-2021-EPI.248}

Objective This cross-sectional analytic study aimed to investigate the situation of work-related diseases and injuries among cultivated agriculturists in the upper northeast of Thailand. The case study of Udon-Thani and Roi-Et provinces is representative of the upper Northeast region of Thailand.

Methods Cases of work-related diseases reported from the health database (HDC) in 2014-2016 among farmers by occupation code were used for analysis. The number of registered farmers from the agricultural office of Thailand and the secondary data in 43 files of occupational diseases from the provincial public health office were collected from 2014 to 2016. The annual morbidity rate of occupational diseases was analyzed and presented in the rate per 100,000 registered farmers.

Results Chronic lung diseases were the first ranking of disease recorded in HDC among visited farmers for health care in the northeast of Thailand. The following rate of diseases in orders were work-related musculoskeletal disorders (WMSDs), noise and heat-related diseases, and pesticide toxicity, respectively. Occupational injuries were as high as the WMSDs. The morbidity rate of Udon-Thani was higher compared to Roi-Et province, and closer to the national rate. The morbidity rates were increasing every year during 2014-2016 which, in 2016, were 479.1, 207.7, and 26.5 per 100,000 farmers for WMSDs, noise and heat-related diseases, and pesticide toxicity for Udon-Thani database. Those rates were higher than a report in HDC at least 2 times.

Conclusions Situations of work-related diseases among cultivated agriculturists in Thailand were not reflected in reality at the present as underestimation. Two main causes that made the underestimation rate; firstly, the number of cases were rarely reported of ICD-10 as occupational diseases, and the second was an inaccuracy of the number of registered farmers in the population. In the future, all agriculturists should be registered in the agricultural and health database.

\section{P-248 STRESS STATUS OF HEALTHCARE DRIVERS DURING THE COVID-19 PANDEMIC IN MALAYSIA}

${ }^{1}$ Abdul Aziz Harith, Zuraida Mohamed, Azyyati Mohammad, Kuang Kuay Lim, Nofi Yuliani Dahlan, Shahida Ismail, Naiemy Reffin, Malindawati Mohd Fadzil, Maznieda Mahjom. 'University of Otago Wellington, New Zealand

\subsection{6/OEM-2021-EPI.249}

Introduction The pandemic of COVID-19 has brought a disastrous impact on every single aspect of human life and activities. The economic and health sectors are most affected by restriction on public movement, daily activities, and burden of coronavirus infection through increased infection and hospitalisation rate. Most research focused on front liners but they overlooked ambulance and healthcare drivers.

Objectives To determine the stress status of healthcare drivers in Malaysia and its associated factors during the COVID-19 pandemic.

Methods This cross-sectional study was conducted among randomly selected 163 healthcare drivers in Negeri Sembilan State Health Department, Malaysia, using self-reported validated questionnaires.

Results A majority of healthcare drivers were male (100\%), married $(90.1 \%)$ with their highest education consisting of a high school certificate $(90.1 \%)$. Ethnically, they consisted of Malay (95.7\%), Indian (3.7\%) and Chinese (0.6\%). Three out of ten healthcare drivers were ambulance drivers, while the rest were non ambulance drivers. The prevalence of stress among healthcare drivers was 7.4\% (95\% CI: $3.7,11.7)$. Higher prevalence was found among ambulance drivers compared to non ambulance drivers; $10.6 \%$ and $6.0 \%$ respectively. There was a significant association between stress and smoking status, performing on-call and duration of working hours in a similar unit.

Conclusion The study revealed that there was a low prevalence of stress among healthcare drivers in Malaysia during the pandemic. The reduced life threatening tasks, fewer emergency incidents and lesser assigned tasks throughout the movement control order during the COVID-19 pandemic could have contributed to the low prevalence statistics among the healthcare drivers in Malaysia. In addition, the effort by the Occupational and Environmental Health Unit, Negeri Sembilan State Health Department in providing consistent safety and health training including stress management might have assisted healthcare drivers to cope with the stressed situation both mentally and physically. 